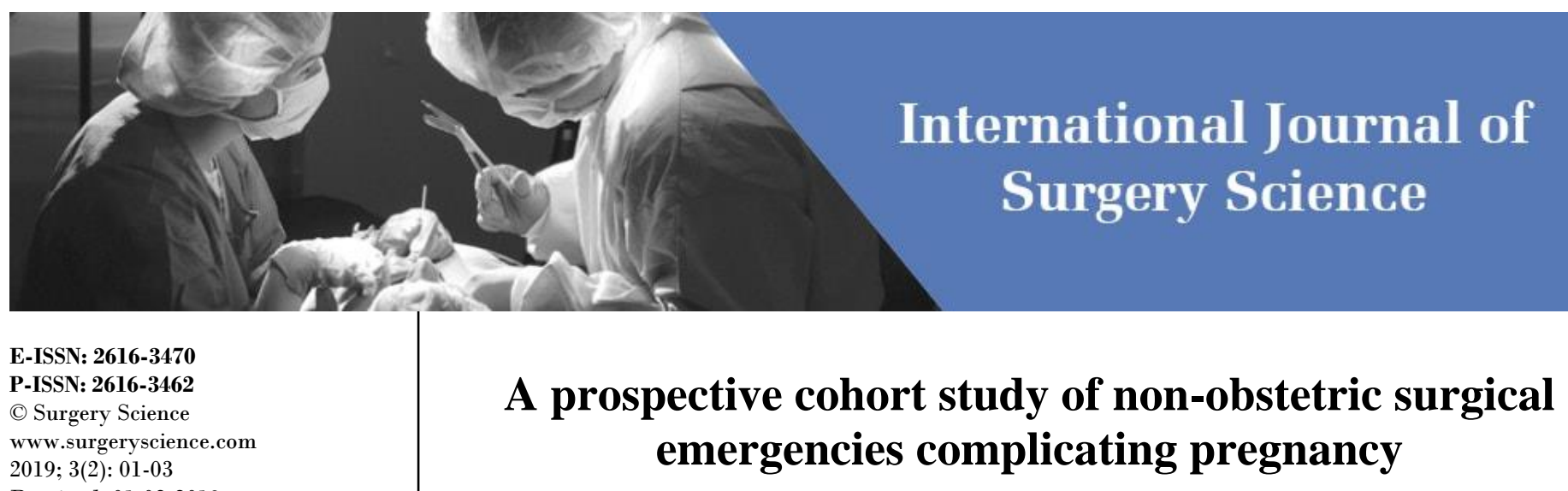

Received: 01-02-2019

Accepted: 05-03-2019

Yassar Arafat

Post Graduate Scholar, Department of Surgery, Government Medical College \& Associated Hospitals, Srinagar Jammu and Kashmir, India

Asim Rafiq Laharwal Lecturer, Department of Surgery, Government Medical College \& Associated Hospitals, Srinagar Jammu and Kashmir, India

\section{Inayat Fatima}

Post Graduate scholar, Department of Gynecology and Obstetrics, SKIMS Medical College, Bemina, Srinagar, Jammu and Kashmir, India

\section{Zubair Gul}

Post Graduate Scholar,

Department of Surgery,

Government Medical College \& Associated Hospitals, Srinagar Jammu and Kashmir, India
Correspondence

Asim Rafiq Laharwal

Lecturer, Department of Surgery,

Government Medical College \&

Associated Hospitals, Srinagar

Jammu and Kashmir, India

\section{Yassar Arafat, Asim Rafiq Laharwal, Inayat Fatima and Zubair Gul}

DOI: https://doi.org/10.33545/surgery.2019.v3.i2a.01

\section{Abstract}

Background: During pregnancy, $0.2 \%$ to $1.0 \%$ of women require general surgery for a non-obstetric problem. The evaluation, work-up, and management of pregnant patients are essential in the practice of emergency medicine to avert maternal and fetal morbidity and mortality. The aims and objectives of this study were to study the common causes of acute abdomen in pregnancy and there management.

Methods: Ours was a prospective cohort study of 50 pregnant patients presenting with a non-obstetric surgical emergency over a period of 1 year in a tertiary care hospital of a developing area.

Results: The mean age of the patients was $27.4 \pm 4.73$ years with most of them being in the age group of 26- 30 years. Majority of patients presented in the $2^{\text {nd }}$ trimester [22 (44\%)]. Ultrasonography was used as the diagnostic modality of choice in $49(98 \%)$ patients. Acute cholecystitis was the most common surgical emergency in our study group accounting for $28 \%$ of all the emergencies, followed by acute appendicitis $(14 \%)$, urinary tract infections (12\%), urolithiasis (12\%) and acute pancreatitis (6\%).

Conclusion: Early suspicion and serial examination in pregnancy may result in appropriate interventions for non-obstetric surgical emergencies. Surgeons should not be hesitant in ordering imaging modalities. Ultrasonography is most common and most useful diagnostic modality in pregnancy.

Keywords: non-obstetric; surgical; pregnancy; emergency; operative

\section{Introduction}

Acute abdomen develops during 1 in 500 pregnancies ${ }^{[1]}$. During pregnancy, $0.2 \%$ to $1.0 \%$ of women require general surgery for a non-obstetric problem ${ }^{[2]}$. The evaluation, work-up, and management of pregnant patients are essential in the practice of emergency medicine. Appendicitis, cholecystitis, and bowel obstruction account for the majority of the abdominal pain syndromes, which require surgical intervention. Evaluation and treatment generally are same as for the non-pregnant patient, but pregnancy may mask some of the typical presenting symptoms, leading to delayed diagnosis. Early suspicion and serial examination in pregnancy may result in appropriate interventions for appendicitis, cholecystitis, pancreatitis and bowel obstruction.

Pregnancy causes many changes that affect the presentation of acute abdominal pain. The gravid uterus, considered an abdominal organ at approximately 12 weeks' gestational age, compresses and displaces the underlying and surrounding viscera ${ }^{[3-5]}$. These changes, combined with a hesitancy to perform radiographic tests, create difficulties in emergency physician's evaluation of pregnant patients ${ }^{[6]}$. Acute abdominal pain can arise from several systems: gastrointestinal, urogenital, gynecologic, or obstetric ${ }^{[7-10]}$. Delay in diagnosis or treatment can cause harm to mother and fetus ${ }^{[1]}$. The aims and objectives of this study were to study the common causes of acute abdomen in pregnancy and there management.

\section{Methods}

Ours was a prospective observational study conducted in the Department of General Surgery, Government Medical College, Srinagar carried out from March 2018 to February 2019. During the study period, 50 pregnant patients presented to the surgical emergency department with nonobstetric surgical emergencies complicating their pregnancy and were studied. On admission, a detailed history was taken from the patient including the presenting complaints with special emphasis towards obstetric history and examination. A Thorough abdominal examination was done in each patient. After doing routine baseline blood investigations, ultrasonography was 
used as the first choice for abdominal imaging. Magnetic Resonance Imaging was used as the second line diagnostic modality when ultrasound was inconclusive. All obstetric emergencies were excluded from the study.

A written and informed consent was taken from the patients for this study. The concerned ethical committee approved the study. The recorded data was compiled and entered in a spreadsheet (Microsoft Excel) and then exported to data editor of SPSS Version 20.0 (IBM Inc., Chicago, Illinois, USA). Continuous variables were expressed as Mean \pm SD and categorical variables were summarized as frequencies and percentages.

\section{Results}

The mean age of the patients was $27.4 \pm 4.73$ years with most of them being in the age group of 26- 30 years. Thirty-five (70\%) patients hailed from the rural areas. Twenty-seven (54\%) patients were primigravida. Majority of patients presented in the $2^{\text {nd }}$ trimester [22 (44\%) patients]. The surgical emergencies complicating pregnancy is shown in Figure 1. Ultrasonography was used as the diagnostic modality of choice in 49 (98\%) patients. Acute cholecystitis was the most common surgical emergency in our study group accounting for $28 \%$ of all the emergencies, followed by acute appendicitis (14\%), urinary tract infections (12\%), urolithiasis (12\%) and acute pancreatitis $(6 \%)$.

\section{Discussion}

Treatment of surgical disease in the gravid patient requires a unique and careful approach where safety of the mother and fetus are both considered. Approaches to diagnosis and therapy of surgical disease in the gravid patient are increasingly clarified and defined in the literature. While in general the principal of diagnosing and treating a pregnant woman with an acute surgical abdominal problem remain the same as those governing the treatment of non-pregnant patient, some important differences are present and can pose problems. As a general rule the condition of mother should always take priority because treatment of surgical diseases in the mother will usually benefit the fetus as well as the mother. Cooperation between a maternal-fetal medicine specialist, surgeon, and obstetrician maximizes the benefits of diagnosis and therapy for the mother and the fetus ${ }^{[7-15]}$.

Acute cholecystitis was the most common surgical emergency and accounted for $28 \%$ of all the surgical emergencies in our study. Cholelithiasis was present in all the cases of acute cholecystitis a finding comparable with the study done by Bouyou $\mathrm{J}$ et al. ${ }^{[16]}$. Most common symptom in acute cholecystitis was pain right upper quadrant, followed by radiation of pain, nausea, dyspepsia and vomiting. Most consistent signs were right upper quadrant tenderness and positive murphy's sign. Conservative management was chosen as the modality of management for acute cholecystitis and consisted of hydration, supportive care and antibiotic therapy.

Acute appendicitis was the second most common surgical emergency in pregnancy in our study and accounted for $12 \%$ of them. Most common symptom in acute appendicitis was pain right abdomen, followed by anorexia, nausea and migration of pain. Most consistent signs were tenderness in right abdomen and rebound tenderness in right iliac fossa. Ultrasound was diagnostic in all the cases in our study, an observation comparable with the study done by Masseli G et al. ${ }^{[17]}$ and Rashid et al. ${ }^{[18]}$. Open appendectomy with a classical McBurney's incision was the chosen modality of treatment in all the cases. There was no post-operative maternal or fetal mortality.
Urinary Tract Infections (UTI) accounted for $12 \%$ of all the surgical emergencies in our study. Patients of UTI usually presented with the symptoms of dull aching pain in the lower abdomen, with history of dysuria and frequency of micturition. Patients had mild tenderness on deep palpation in lower abdomen. Most patients had asymptomatic bacturia with pus cells in urine of 26-40/HPF and cystitis in few of them. Most common micro- organism isolated was Escherechia coli in 5 patients and Proteus mirabilis in 1 patient.

Urolithiasis accounted for $12 \%$ of cases of surgical emergencies in our study. Patients typically presented with colicky pain with radiation and vomiting. Five patients had right-sided renal calculi and 1 patient had left-sided renal calculus. All the patients had stones less then $1 \mathrm{~cm}$. All the 6 patients were successfully managed conservatively with hydration, analgesics and antibiotics and with expulsive therapy. No surgical intervention was required in any patient.

Acute pancreatitis accounted for $6 \%$ of cases in our study. All the cases were having gall stone induced acute pancreatitis. Trans abdominal ultrasonography was confirmatory in diagnosing all the patients showing an edematous, enlarged pancreas. All the patients were managed medically and responded successfully.

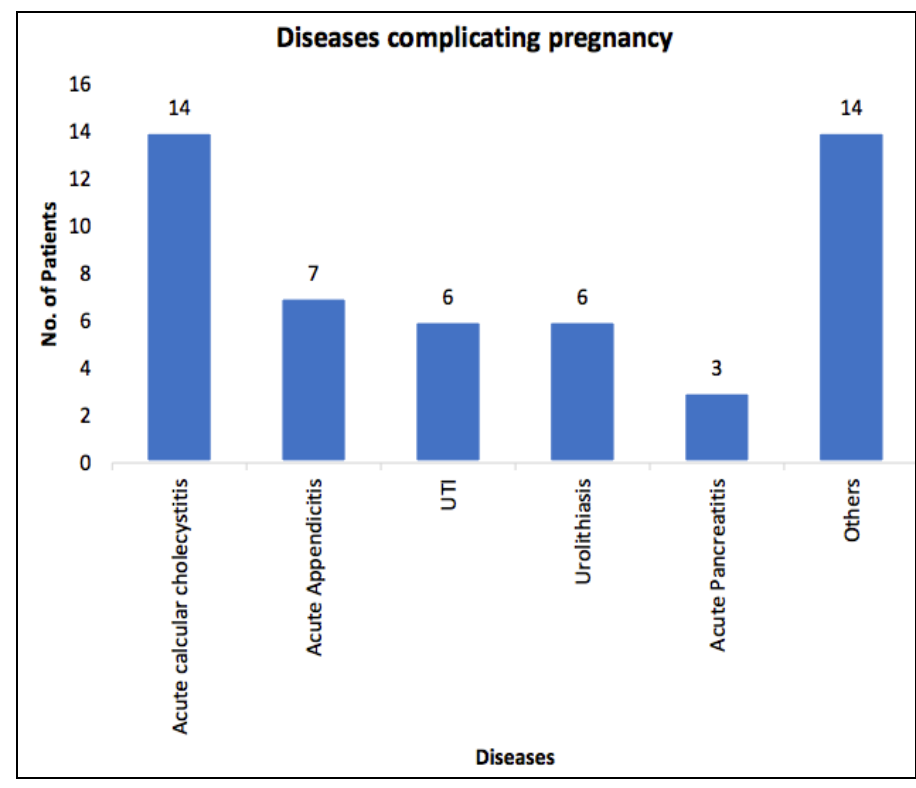

Fig 1: Surgical emergencies complicating pregnancy.

\section{Conclusion}

Abdominal pain and various surgical emergencies are a common cause of anxiety-provoking consultations in pregnancy. Subtle differences in laboratory values need to be acknowledged and atypical presentations of common diseases can be seen during pregnancy. Surgeons should not be hesitant in ordering imaging modalities. Ultrasonography is most common and most useful diagnostic modality in pregnancy.

\section{References}

1. Coleman MT, Trianfo VA, Rund DA. Nonobstetric emergenciesin pregnancy: trauma and surgical conditions. Am J Obstet Gynecol. 1997; 177(3):497-502.

2. Parangi S. Surgical gastrointestinal disorders during pregnancy. Am J Surg. 2007; 193(2):223-32.

3. Firstenberg MS, Malangoni MA. Gastrointestinal surgery during pregnancy. Gastroenterol Clin North Am. 1998; 27(1):73-88. 
4. Kilpatrick CC, Monga M. Approach to the acute abdomen in pregnancy. Obstet Gynecol Clin North Am. 2007; 34(3):389-402.

5. Oto A. MR imaging in the triage of pregnant patients with acute abdominal and pelvic pain. Abdom Imaging. 2009; 34(2):243-50.

6. Fan SJ, Xiang JX, Xiao M, Wang FH, Lin XJ, Zhou XH et al. Influence of acute pancreatitis in pregnancy on pregnancy outcomes and neonates. Zhongguo Dang Dai Er Ke Za Zhi. 2018; 20(4):274-278.

7. Singh AK, Desai H, Novelline RA. Emergency MRI of acute pelvic pain: MR protocol with no oral contrast. Emerg Radiol. 2009; 16(2):133-41.

8. Vissers RJ, Lennarz WB. Pitfalls in appendicitis. Emerg Med Clin North Am. 2010; 28(1):103-18.

9. Szweda H, Jóźwik M. Urinary tract infections during pregnancy - an updated overview. Developmental Period Medicine. 2016; XX:42-63.

10. Augustin G. Surgical Emergencies During Pregnancy. In: Di Saverio S., Catena F., Ansaloni L., Coccolini F., Velmahos G. (eds) Acute Care Surgery Handbook. Springer, 2017, 485-507.

11. El-Messidi A, Alsarraj G, Czuzoj-Shulman N, Mishkin DS, Abenhaim HA. Evaluation of management and surgical outcomes in pregnancies complicated by acute cholecystitis. J Perinat Med. 2018; 46(9):998-1003.

12. Mittal P, Wing DA. Urinary Tract Infections in Pregnancy. Clin Perinatol. 2005; 32:749-764.

13. Connolly A, Thorp JM. Urinary tract infections in pregnancy. Urol Clin North Am. 1999; 26(4):779-87.

14. Ovalle A, Levancini M. Urinary tract infections in pregnancy. Curr Opin Urol. 2001; 11(1):55-9.

15. Hooton TM. Fluoroquinolones and resistance in the treatment of uncomplicated urinary tract infection. Int $\mathbf{J}$ Antimicrob Agents. 2003; 22(2):65-72.

16. Bouyou J, Gaujoux S, Marcellin L, Leconte M, Goffinet F, Chapron $\mathrm{C}$ et al. Abdominal emergencies during pregnancy. Journal of Visceral Surgery Dec. 2015; 152(6):S105-S115.

17. Masselli G, Derchi L, McHugo J et al. Acute abdominal and pelvic pain in pregnancy: ESUR recommendations. Eur Radiol. 2013; 23(12):3485-500.

18. Rashid A, Nazir S, Kakroo SM, Chalkoo MA, Razvi SA, Wani AA. Laparoscopic interval appendectomy versus open interval appendectomy: A prospective randomized controlled trial. Surg Laparosc Endosc Percutan Tech. 2013; 23(1):93-6. 